\title{
Editorial
}

\section{Hand Hygiene in the New Millennium: Drawing the Distinction Between Efficacy and Effectiveness}

\author{
L. Clifford McDonald, MD
}

Recently, much attention has been focused on hand hygiene in health care with the publication of a new national guideline. ${ }^{1}$ In the guideline, washing hands with soap and water is replaced by rubbing hands with an alcohol hand rub as the primary means of hand hygiene to be used by healthcare personnel involved in routine patient care. The rationale behind this shift is the documented increased antimicrobial efficacy of alcohol hand rubs over washing hands with either plain soap and water or an antimicrobial soap. In addition, there is the potential for increased compliance with hand hygiene because hand rubbing requires less time, results in less skin irritation, and does not require proximity to a sink. The only caveat is that the hands must be free from visible soiling prior to the use of an alcohol hand rub. ${ }^{1}$ Two articles appearing in this issue of Infection Control and Hospital Epidemiology raise important issues pertaining to the position of alcohol hand rubs in the new guideline.,3

The first of these, by Dharan et al., describes a comparison of the antimicrobial efficacies of several waterless hand hygiene products when used at short application times (15 seconds). ${ }^{2}$ These investigators compared one gel containing $60 \%$ ethanol with four alcohol-based rinses including a reference rinse containing $60 \%$ isopropyl alcohol in a crossover study of 12 volunteers in whom they tested the five hand rubs according to a modification of the European standard for establishing the efficacy of alcohol hand rubs (EN 1500). Each test involved determining the $\log$ reduction of introduced bacterial contamination on the fingertips of one hand cleaned with the test gel or rinse compared with the other hand cleaned with the reference rinse (60\% isopropyl alcohol).

The main results of this study were that all hand rinses satisfied the EN 1500 criteria for efficacy as defined by having log bacterial load reductions that were either statis- tically similar to or superior to the load reduction observed using the reference rinse (60\% isopropyl alcohol). ${ }^{2}$ However, the reduction observed with the gel was consistently approximately $1 \log$ less than that observed with the reference rinse and this difference was statistically significant $(P<.025)$; thus, the gel failed to meet the European standard for efficacy when used for 30 or 15 seconds. In this regard, the findings of Dharan et al. confirm the results of the previous study by Kramer et al. in which none of ten different gel formations met the EN 1500 criteria using a 30-second cleansing period. ${ }^{4}$ Dharan et al. conclude that in testing performed "under stringent conditions similar to clinical practice," rinses are more efficacious than alcoholbased gels. ${ }^{2}$

In the second of these, Mody et al. attempt to measure the impact of an alcohol-based hand rub $(62 \%$ ethanol gel) on compliance with hand hygiene in a long-term-care facility. ${ }^{3}$ This was a four-phase interventional trial (ie, baseline, education, introduction of the alcohol rub, and longterm follow-up of the rub) conducted on two 36-bed wards of a long-term-care facility. On ward $A$, the rub was made available along with usual soap and water hand washing. On ward B, only soap and water hand washing was available. No important difference was found in any outcome measure between the two wards at baseline, and there were no differences between baseline measurements and measurements after the educational intervention in either of the two wards. Following the introduction of the alcohol rub, however, staff on ward A were more likely to agree that the rub was more convenient and faster than soap and water and were more likely to disagree that the alcohol rub was more drying than soap and water. Likewise, staff on ward A self-reported an increased frequency of hand hygiene following introduction of the rub when compared with baseline or with ward $B$. No differences were 
observed between the two wards in the degree of hand colonization with gram-negative bacilli, Candida species, Staphylococcus aureus, or vancomycin-resistant enterococ$\mathrm{ci}$, either at baseline or following the educational intervention or introduction of the hand rub. However, the hand rub was more effective than plain soap and water in removing gram-negative bacilli and $S$. aureus. No differences were observed in the number of nosocomial infections between the two wards either at baseline or following either the educational intervention or the introduction of the alcohol rub.

Before we jump to the conclusion that the results of these two studies suggest that alcohol rub does not work, we must draw a distinction between efficacy and effectiveness and realize the limitations of these studies. Neither study was designed well enough to demonstrate the effectiveness, in practice, of a hand rub (rinse or gel) in preventing either nosocomial infections or the spread of nosocomial pathogens among patients. For example, the study by Dharan et al. simply compared the antimicrobial efficacies of different hand rub formulations under controlled test conditions. ${ }^{2}$

In contrast, the study by Mody et al. did attempt to measure the effectiveness of hand rub over plain soap and water in preventing infections. ${ }^{3}$ However, because infections were compared between only two 36-bed units during 11 months, the study had low statistical power to demonstrate anything but a major reduction in rates. This fact led to the authors' recommendation that a larger, multicenter study be undertaken. Moreover, nosocomial infections are associated with a variety of patient risk factors, from use of invasive devices to underlying severity of illness, which may be unrelated to the adequacy of hand hygiene among healthcare workers. In contrast, the incidence of transmission of a typical nosocomial pathogen, such as that defined by the new detection of methicillin-resistant $S$. aureus (MRSA) in either screening or clinical cultures, is more directly the result of transient hand carriage and may be a better, more sensitive indicator of the effectiveness of hand hygiene.

In addition, Mody et al. tried to detect an impact of alcohol rub by determining the degree of microbial colonization of healthcare workers' hands based on unannounced cultures. ${ }^{3}$ Although this would have been a reasonable surrogate marker for effectiveness, again no difference could be documented between intervention (alcohol rub) and control (plain soap and water) wards. Similar to the multitude of studies reviewed in the new guideline, ${ }^{1}$ Mody et al. were able to demonstrate the superior efficacy of alcohol rub over plain soap and water based on results of their cultures. ${ }^{3}$ Nonetheless, their culture methodology was technically limited because they performed an overnight incubation of the initial broth media used for sampling, which made it difficult to determine the relative microbial contamination of healthcare workers' hands.

Transient hand colonization is generally accepted as the primary means of cross-transmission and subsequent infection in hospitals. ${ }^{1}$ Thus, the stage is set for effective hand hygiene to have a major impact on healthcare out- comes. However, there remain two prerequisites for hand hygiene to be effective in reducing cross-transmission. First, the method of hand hygiene must be efficacious in reducing or eliminating transient hand colonization. Second, there must be good compliance with hand hygiene. The relative importance of compliance versus efficacy in the overall effectiveness of hand hygiene has not been well studied.

What we do know from the study by Dharan et al., ${ }^{2}$ as well as from other similar studies, ${ }^{4}$ is the current upper limit of achievable efficacy using an alcohol hand rub, whether gel or rinse. We also know from a variety of sources that current levels of compliance with hand hygiene in most hospitals are deplorable, with rates hovering around $40 \%$ or less. ${ }^{1}$ Given such poor compliance, most of us working in infection control would emphasize improving compliance over a small improvement in efficacy. Nonetheless, the findings of Dharan et al. may aptly argue that there is room for an improvement in efficacy as well as compliance. The question remains whether a small, albeit statistically significant, difference in efficacy between rinses and gels is clinically significant and, if it is, whether a benefit of gels over rinses in terms of improved skin tolerability may lead to increased compliance that would overshadow their slight antimicrobial inferiority.

Several methods are used to determine the microbial efficacy of alcohol hand rubs. ${ }^{1}$ The method used by Dharan et al. was a variation of the European standard (EN 1500) for establishing the efficacy of an alcohol hand rub, which is based on comparison against a reference standard of $60 \%$ isopropyl alcohol hand rinse in reducing an introduced microbial load. According to this standard, a tested product is considered non-efficacious if it is statistically inferior in terms of reducing microbial load when compared with the reference hand rinse.

Others have pointed out that the definition of efficacy based on the activity of $60 \%$ isopropyl alcohol is arbitrary. ${ }^{5}$ Although there are some data linking the log reduction attained by $60 \%$ isopropyl rinse with effectiveness, these are limited to the results of the study by Pittet et al. in which the introduction of a multifaceted hand hygiene program including $60 \%$ isopropyl alcohol hand rinse resulted in a reduction of transmission of MRSA. ${ }^{6}$ It remains unclear from that study, however, whether increased compliance with hand hygiene (from $48 \%$ to $66 \%$ ) played the more important role in effectiveness than increased antimicrobial efficacy of the alcohol hand rub. Moreover, the use of an historical control design limited that study because the introduction of other new MRSA control measures may have impacted transmission.?

In contrast to the situation in Europe, in the United States the standard for establishing the efficacy of alcohol hand rubs is based on the Food and Drug Administration's Tentative Final Monograph (TFM). ${ }^{1}$ The TFM test method, similar to the EN 1500, involves first contaminating the hands with an indicator organism and then performing baseline cultures and cultures after hand hygiene is performed using the test formulation. The TFM criteria for 
efficacy, however, consist of a 2-log reduction of the indicator organism on each hand within 5 minutes of the first use and a 3-log reduction within 5 minutes after the tenth use; there are no comparisons made to the log reduction achieved using a reference hand rub.

Although the TFM criteria, similar to the EN 1500 criteria, have never been correlated with clinical effectiveness, the log reduction required by the TFM criteria may better reflect the realities of microbial hand contamination that commonly occurs in clinical practice. Several investigators have studied hand contamination with potential nosocomial pathogens during routine patient care. ${ }^{811}$ The results of studies involving the random sampling of healthcare workers during their work shifts (ie, without any assurance that hand hygiene had been performed any time recently) suggest that under certain circumstances the entire hands of healthcare workers may become colonized with as many as $10^{6}$ colony-forming units (CFU) of potential pathogens..$^{10}$ However, studies focusing on the degree of contamination resulting from a single "episode" of patient care generally indicate $10^{3} \mathrm{CFU}$ to be the maximum microbial burden that an alcohol hand rub should be expected to eradicate. ${ }^{8,9,11}$ In one study, the microbial load on the five fingertips of one hand was as high as 300 CFU following certain high-risk patient care activities; however, the median degree of contamination following the care of any individual patient was only 39 CFU. ${ }^{11}$ In contrast, the microbial load introduced to the fingertips of volunteers in the study by Dharan et al. was at least $10^{7}$ and rubs were deemed non-efficacious according to the EN 1500 criteria because they were able to reduce microbial counts by "only" approximately $10^{4} .^{2}$ Therefore, based on what is known about the degree of contamination that commonly occurs in clinical practice, it appears that the EN 1500 criteria may be excessively stringent.

Certainly if all other things were equal, we should embrace the alcohol hand rub with greatest efficacy. The attractiveness of gel formulations, however, is their potential to be better accepted by healthcare workers due to decreased skin irritation compared with rinses. The article by Mody et al. in this issue, ${ }^{3}$ as well as other articles, ${ }^{1}$ highlight the reduction in skin irritation associated with the use of alcohol hand gels over plain soap and water. Although independent studies directly comparing the acceptability of rinses with the acceptability of gels may be lacking, it is the perception of many, 5,12-14 including this author, that gels are better tolerated than most rinses. Moreover, there exists at least anecdotal evidence to suggest that replacement of an alcohol hand rinse with a gel can increase compliance with hand hygiene. ${ }^{14}$ Thus, there is good reason to be careful not to "raise the flag of concern" too high over the slight antimicrobial inferiority of gels compared with rinses as this may unnecessarily undermine our efforts to introduce and gain acceptance for alcohol hand rubs in general.
A final point made by both Dharan et al. ${ }^{2}$ and Mody et $a .^{3}$ is something with which we can all agree: the need for future multicenter, controlled studies to document and quantify the effectiveness of hand rub formulations and hand hygiene-related behavior modification techniques for decreasing transmission of nosocomial pathogens. Such studies should include aspects of the study by Mody et al. in terms of a prospective control unit or ward, rather than relying on historical controls. Future studies should also include appropriate microbial sampling as a surrogate indicator of effectiveness. In addition, such studies should include a more stringent assessment of compliance than the self-reports of hand hygiene used by Mody et al. Just as Pittet et al. did in their study of effectiveness, ${ }^{6}$ compliance is probably best measured via periodic direct observational surveys, supplemented by a measurement of the amount of hand hygiene product consumed by healthcare workers. For reasons already mentioned, transmission of a typical nosocomial pathogen, such as MRSA, may serve as a better outcome indicator for effectiveness. Finally, it could be reasonably hoped that, in addition to documenting and quantifying the effectiveness of one or more formulations of alcohol hand rub, results of such prospective, multicenter studies would increase the recognition of hand hygiene as one of the most important interventions to prevent adverse outcomes in health care.

\section{REFERENCES}

1. Boyce JM, Pittet D. Guideline for hand hygiene in health-care settings: recommendations of the Healthcare Infection Control Practices Advisory Committee and the HICPAC/SHEA/APIC/IDSA Hand Hygiene Task Force. Infect Control Hosp Epidemiol 2002;23(suppl):S3S40.

2. Dharan S, Hugonnet S, Sax H, Pittet D. Comparison of waterless hand antisepsis agents at short application times: raising the flag of concern. Infect Control Hosp Epidemiol 2003;24:160-164.

3. Mody L, MeNeil SA, Sun R, Bradley SF, Kauffman CA. Introduction of a waterless alcohol-based hand rub in a long-term-care facility. Infect Control Hosp Epidemiol 2003;24:165-171.

4. Kramer A, Rudolph P, Kampf G, Pittet D. Limited efficacy of alcoholbased hand gels. Lancet 2002;359:1489-1490.

5. Boyce JM, Larson EL, Weinstein RA. Alcohol-based hand gels and hand hygiene in hospitals. Lancet 2002;360:1509-1510.

6. Pittet D, Hugonnet S, Harbarth S, et al. Effectiveness of a hospital-wide programme to improve compliance with hand hygiene: infection control programme. Lancet 2000;356:1307-1312.

7. Stone S, Teare L, Cookson B. Guiding hands of our teachers: Handhygiene Liaison Group. Lancet 2001;357:479-480.

8. Casewell M, Phillips I. Hands as route of transmission for Klebsiella species. BMJ 1977;2:1315-1317.

9. Fox MK, Langner SB, Wells RW. How good are hand washing practices? Am J Nurs 1974;74:1676-1678.

10. Ayliffe GA, Babb JR, Davies JG, Lilly HA. Hand disinfection: a comparison of various agents in laboratory and ward studies. I Hosp Infect 1988;11:226-243.

11. Pittet D, Dharan S, Touveneau S, Sauvan V, Perneger TV. Bacterial contamination of the hands of hospital staff during routine patient care. Arch Intern Med 1999:159:821-826.

12. Hoffman P, Cookson B, Teare L. Alcohol-based hand gels and hand hygiene in hospitals. Lancet 2002;360:1510.

13. Diekema DJ. Alcohol-based hand gels and hand hygiene in hospitals. Lancet 2002;360:1510.

14. Girard R, Aho LS, Goetz ML, Labadie JC, Lejeune B. Alcohol-based hand gels and hand hygiene in hospitals. Lancet 2002;360:1510-1511. 